\title{
BMJ Open Radiotherapy for head and neck tumours using an oral fixation and parameter acquisition device and TOMO technology: a randomised controlled study
}

\author{
Xiaofang Zhang (D) , ${ }^{1}$ Tianlu Wang, ${ }^{1}$ Xinyan Xiao, ${ }^{2}$ Xia Li, ${ }^{1}$ Chen Yu Wang, ${ }^{3}$ \\ Bo Huang, ${ }^{4}$ Lei $\mathrm{He},{ }^{5}$ Yingqiu Song ${ }^{1}$
}

To cite: Zhang $X$, Wang $T$, Xiao X, et al. Radiotherapy for head and neck tumours using an oral fixation and parameter acquisition device and TOMO technology: a randomised controlled study. BMJ Open 2021;11:e052542. doi:10.1136/ bmjopen-2021-052542

- Prepublication history and additional supplemental material for this paper are available online. To view these files, please visit the journal online (http://dx.doi.org/10.1136/ bmjopen-2021-052542).

Received 21 April 2021 Accepted 19 October 2021

Check for updates

(C) Author(s) (or their employer(s)) 2021. Re-use permitted under CC BY-NC. No commercial re-use. See rights and permissions. Published by BMJ.

For numbered affiliations see end of article.

Correspondence to Dr Yingqiu Song; syq18900917411@163.com

\section{ABSTRACT}

Introduction Radiotherapy has become one of the main methods used for the treatment of malignant tumours of the head and neck. Spiral tomographic intensitymodulated radiotherapy has the many advantages of precision radiotherapy, which puts forward high requirements for postural reproducibility and accuracy. We will aim to ensure that the accurate positioning of the tumour will reduce the side effects of radiotherapy caused by positioning errors. We will design and implement this clinical trial using the patent of 'a radiotherapy oral fixation and parameter acquisition device (patent number: ZL201921877986.5)'.

Methods and analysis This will be a randomised, controlled, prospective study with 120 patients with head and neck tumours. Using the random number table method, a random number sequence will be generated, and the patients will be enrolled in the experimental group (oral fixation device) and the control group (conventional fixation) in a 2:1 ratio. The primary outcome will be the progression-free survival time after the treatment. Secondary outcomes will include the oral mucosal reaction and the quality of life. Follow-ups will be carried out according to the plan. This is V.1.0 of protocol on 1 April 2021. The recruitment process for this clinical trial commenced on 1 May 2021, and will end on 1 October 2022.

Ethics and dissemination The trial received ethical approval from Medical Ethics Committee of Liaoning Provincial Cancer Hospital (number 20210131X). The final results will be presented at a scientific conference and published in a peer-reviewed journal in accordance with the journal's guidelines.

Trial registration number ChiCTR2100045096.

\section{INTRODUCTION}

The incidence of head and neck tumours is increasing annually, accounting for about $10 \%$ of all malignant tumours. ${ }^{1}$ Malignant tumours of the head and neck are moderately sensitive to radiation and radiotherapy has become one of the main treatment
Strengths and limitations of this study

- This patient-based randomised controlled study will involve a sufficient number of patients using an oral fixation and parameter acquisition device for 2 years.

- This study may put forth a treatment standard based on oral fixation and parameter acquisition device and tomotherapy system technology.

- The comparison of the efficacy of the treatments for head and neck tumours may bridge the gap between mouthpiece-tomotherapy and conventional tomotherapy.

- This study may be limited by patient heterogeneity and the loss of follow-up data that may compromise the availability of the data.

methods. After treatment, the 5-year survival rate of patients with malignant tumours of the head and neck can reach approximately $50 \% .^{2}$ Precision radiotherapy includes traditional three-dimensional (3D) conformal radiotherapy and intensity-modulated radiotherapy (IMRT). ${ }^{3}$ Spiral tomographic intensity-modulated radiation therapy (tomotherapy system, TOMO) is a novel method in the field of IMRT technology, which uses a $360^{\circ}$ spiral radiation delivery system similar to a spiral CT scan. ${ }^{4}$ Compared with the traditional fixed-field IMRT, TOMO has the advantage of using more independent directions of rays, which can better adapt to the target dose. ${ }^{5}$ By quickly turning on and off the blades in the rotating collimator, TOMO can maintain the radiation dose in the complexshaped tumour area while reducing the radiation exposure to the at risk surrounding organs and tissues. ${ }^{6}$ For the protection of radiotherapy efficacy, reproducibility and the accuracy of radiotherapy and body position are becoming increasingly important. ${ }^{7}$ If the 
reproducibility of the posture is poor, the isocentre of the actual treatment shifts from the planned isocentre, making the actual dose distribution and the treatment plan inconsistent, resulting in an insufficient tumour dose and the use of an excessive organ-endangering dose. Compared with conventional radiotherapy, this situation results in greater side damage. ${ }^{89}$

Poor postural reproducibility, one of the reasons for oral mucositis, is a common adverse reaction in radiotherapy, with an incidence of more than $40 \% \cdot{ }^{10-12}$ Clinical symptoms such as dry mouth, dry throat, dysphagia, dysgeusia, oral ulcers, decreased appetite, mucosal erythema, congestion, oedema, erosion and pain will appear in the patient's mouth, ${ }^{13}$ inducing bacterial and viral infections, oral bleeding, aggravated mucosal damage, local pain, difficulty in eating, severe oral pain, refusal to eat, ${ }^{14}$ weight loss, decreased immune function, decreased treatment tolerance, hindered progress of radiotherapy and a prolonged course of treatment, leading to a decrease in the rate of local tumour control. ${ }^{3}$ Therefore, it is important to ensure the consistency of each radiotherapy position when performing radiotherapy for head and neck tumours based on TOMO technology. ${ }^{15}$ In order to ensure the accurate positioning of the tumour, the patient's jaw bone should be fixed before radiotherapy to ensure the implementation of precise and efficacious treatment. ${ }^{16}$ Therefore, to ensure the repeatability and accuracy of the radiotherapy position during TOMO treatment, and to ensure the timeous monitoring of the oral mucosa and glandular secretion status of patients with the head and neck tumour, the applicant designed a clinical trial based on the patent of 'a radiotherapy oral fixation and parameter collection device' (Patent Number: ZL201921877986.5). In future, we will formulate intervention methods and improve the corresponding diagnosis and treatment plans based on the results of this clinical trial. This clinical trial is expected to further improve the efficacy of radiotherapy and improve the quality of life (QoL) and survival of patients.

\section{METHODS AND ANALYSIS \\ Design}

This clinical trial will be conducted at the Medical Centre of Liaoning Cancer Hospital (Tertiary Medical Centre) in China. This trial is a prospective study, using the TOMO treatment device to study the safety and effectiveness of radiotherapy in patients with cancer of the head and neck tumours, ${ }^{17}$ involving 120 enrolled patients. Using the random number table method, a random number sequence will be generated, and the patients will be enrolled in the experimental group and the control group in a 2:1 ratio. The experimental group will use the radiotherapy oral fixation and parameter collection device (Patent Number: ZL201921877986.5) while the control group will not. This study will not use a blinding process for the trial participants, because the treatment process enable a clear differentiation between the experimental group and the control group.

The trial sponsors are the Cancer Research Programme of National Cancer Center (NCC2017A08) and Shenyang Major Scientific Research Projects (No. 191124090). This is V.1.0 of protocol on 1 April 2021. The recruitment process for this clinical trial commenced on 1 May 2021, and will end on 1 October 2022.

\section{Patient and public involvement}

The public is not involved in the design of this study. The results of the study will be presented at a conference or academic journal, and the public will not be involved in the dissemination plan of the study findings during this process.

\section{Study participants}

The patient's treating physician obtains the patient's consent and explains the trial to the patient who meet the enrolment criteria. The computer will automatically generate the allocation sequence using the random number table method. The team's researchers will recruit participants and assign interventions to them. Implementing the sequence of allocation is the order of sequential numbers, opaque, sealed and researchers who are in contact with participants before dispensing interventions will not know in advance.

This study will include 120 adult patients meet the inclusion criteria and who use radiotherapy. The inclusion and exclusion criteria are listed in table 1.

\section{Sample size}

It will be necessary to evaluate whether there is a difference between the two groups. ${ }^{18}$ The sample size of the experimental group will be set as $n_{1}$ and the sample size of the control group as $n_{2}, n_{1} n_{2}=1: k, k=2$. The two groups will have different sample sizes, therefore, a two-sided test

\section{Table 1 Subject inclusion and exclusion criteria}

\begin{tabular}{ll}
\hline Inclusion criteria & Exclusion criteria \\
\hline Patients with malignant tumours of the head and neck. & Patients with liver and kidney dysfunction. \\
No brainstem/medulla oblongata invasion. & Bone marrow suppression above grade three. \\
KPS $\geq 70$ points. & Moderate to severe cardiac insufficiency. \\
& No vital organ failure.
\end{tabular}

KPS, Karnofsky Performance Status. 
will be used used. ${ }^{19}{ }^{20}$ The sample size calculation formula will be:

$$
n_{1}=\frac{k+1}{k}\left[\frac{\left(z_{\alpha / 2}+z_{\beta}\right) \sigma}{\delta}\right]^{2}
$$

Where $\sigma$ will be the overall SD, estimated to be $1.84, \delta$ will be the difference between the two sets of numerical variables, estimated to be $1, Z_{\alpha}$ will be the standard normal value corresponding to the inspection level $\alpha, Z_{\beta}$ will be the standard normal value corresponding to $\beta$, If $\sigma=1.84$ months, $\delta=1$ month, $\alpha=0.05, \beta=0.20, \mathrm{Z}_{\alpha / 2}=\mathrm{Z}_{0.05 / 2}=1.96$, and $\mathrm{Z}_{\beta}=\mathrm{Z}_{0.20}=0.842$, then by substituting the above formula will give:

$$
\begin{gathered}
n_{1}=\frac{2+1}{2}\left[\frac{(1.96+0.842) \times 1.84}{1}\right]^{2}=39.9 \approx 40 \\
n_{2}=2 n_{1}=80
\end{gathered}
$$

That is, 40 cases will be in the control group and 80 cases in the experimental group, with the total sample size being 120 cases.

\section{Primary study outcome}

Progression-free survival time

The primary outcome will be the progression-free survival time (PFS) time after radiotherapy, which refers to the number of months between the initiation of radiotherapy and the first evidence of progressive disease, as defined by the Response Evaluation Criteria In Solid Tumours. ${ }^{21}$

\section{Secondary study outcomes}

Oral mucosal reaction

Oral mucosal reactions will be assessed once a week and will include both objective and subjective assessments. The objective assessment will include the objective grading of the radiation oral mucositis using the Common Terminology Criteria for Adverse Events (CTCAE) V4.0 and the Oral Mucosal Assessment Scale ulcer score. ${ }^{22}$ The subjective assessment will include the WHO Oral Mucositis Score, as well as the Mouth and Throat Pain (MTS) Problem Score.
Quality of life

QoL is counted by The QoL Questionnaire, which is statistically evaluated every 2 weeks. The QoL questionnaire consist of three parts: The European Organization for Reasearch and Treatment of Cancer (EORTC) Quality of Life Questionnaire-Core 30 (QLQ-C30) V3.0, ${ }^{23}$ oral and throat pain questions, and WHO oral mucositis score questions, for a total of 32 questions. The details can be viewed in online supplemental material.

\section{Study outline}

Equipment and technical support

A 3D treatment planning system (3D-TPS), TPS, 3D water tank, linear electron accelerators, image fusion software, TOMO, medical linear accelerators and CT will be provided in the radiotherapy department. Equipment and technical support details are presented in table 2 .

\section{CT localisation scanning method}

The patient will lie supine on a special positioning neck and shoulder stand for radiotherapy, the polymer low-temperature hydrolyzed plastic neck and shoulder membrane will be fixed in position, and the laser light will be positioned on the midline and both sides of the head (for a dedicated external positioning system), on the neck and shoulder membrane and body surface. The corresponding reference point will be marked with the placements of lead pellets as a mark. ${ }^{24}$ A spiral CT will scan the whole brain continuously with a pitch of $2 \mathrm{~mm}$ under calm breathing. The data will be transmitted to the ADAC Pinnacle3 Treatment Plan System (TPS) workstation via the network, and then MRI will be collected in 2D. An attenuation correction of the MRI image will be performed with the CT data, and the image will be reconstructed using the iterative method. ${ }^{25}$

\section{Formulation and implementation of radiotherapy plans}

An attending physician in the radiotherapy department and an experienced diagnostic imaging physician will delineate the radiotherapy target area on the MRI and CT fusion images. Tumour area GTV (gross tumour volume),

\begin{tabular}{llll}
\hline Table 2 & Summary of the new technology project equipment & & \\
\hline Device & Device model & Manufacturer & Factory no \\
\hline 3D-TPS & Pinnade & Philips & 20050628 \\
TPS & Pinnacle Smart Enterprise & Philips & 20030126 \\
3D water tank & RFA-300 & IBA & 20021210 \\
Linear electron accelerator & IX6117 & Varian & 20151217 \\
Image fusion software & MIM & MIM Software & 20151125 \\
Linear electron accelerator & UNIQUE & Varian & 20140122 \\
TOMO & Tomotherapy HD & Accuracy & 20151125 \\
Medical linear accelerators & Clinac IX & Varian & 20151203 \\
CT & SOMATOM & Siemens & 20140731 \\
\hline
\end{tabular}

3D water tank, three-dimensional (3D) scanner water tank system; TOMO, tomotherapy system; TPS, treatment planning system. 
clinical target area CTV (clinical tumour volume), CTV will be placed $0.3 \mathrm{~cm}$ outside as the planning target area PTV (planning tumour volume), delineating the patient's brain stem, spinal cord, mandible, inner ear of the temporomandibular joint. For important functional areas such as crystals, TOMO radiotherapy plans are developed on the Pinnacle3 treatment planning system; VARIAN' s high-energy electron beam accelerator 6-MV X rays are used for TOMO irradiation. ${ }^{26}$

\section{Treatment plan}

Patients who meet the enrolment criteria will be treated with radiotherapy on the basis of protecting important functional areas such as the brain stem, spinal cord, parotid gland, mandible, temporomandibular joint, inner ear and lens. The dose of radiotherapy in the tumour area will be 6996-7392 cGy/33F, in high-risk areas, the dose will be $6006 \mathrm{cGy} / 33 \mathrm{~F}$, and in low-risk areas the dose will be $5096 \mathrm{cGy} / 28 \mathrm{~F}$, five times a week. The experimental group will use radiotherapy oral fixation and parameter collection devices, while the control group will not.

\section{Post-IMRT-TOM0 follow-up}

After radiotherapy, patients will be reviewed every 3 months for 6 months to evaluate whether they have recurrence and oral function. After 6 months to 2 years after treatment, a recheck will be performed every 6 months. ${ }^{27}$ The examination will include: oral enhanced magnetic resonance, cervical lymph node colour Doppler ultrasound, routine blood tests and liver and kidney function tests, to evaluate the effectiveness of the TOMO radiotherapy (with or without oral fixation and parameter acquisition devices) according to the standards set by the committee. The follow-up period for PFS is 2 years. In addition, We make assessment of Oral mucosal reaction and QoL at immediate post-radiotherapy (post-RT), 2 weeks post-RT, 1 month, 3 months, 6 months and 2 year post-RT of QoL. The research outline is shown in figure 1 .

\section{Data analysis}

After the final patient completes the follow-up study, the committee will assess the occurrence of the primary and secondary endpoints. SPSS V.25.0 software will be used for the data collation and analysis, and in all the statistical tests $\mathrm{p}<0.05$ will be considered as a statistically significant difference. Survival time will be defined from the date of diagnosis of head and neck tumours to the date of death or the date of the most recent follow-up. The overall survival and PFS will be calculated using the Kaplan-Meier method; the Cox regression method will be used to calculate the HR and its $95 \% \mathrm{CI}$, and the $\chi^{2}$ test will be used to analyse death and the difference between the different variables in surviving patients. ${ }^{28}$ The QoL at 6 months will be scored by Functional Assessment of Cancer Therapy: General (FACT-G). The $\chi^{2}$ test, combined with the propensity matching analysis method will be used to eliminate to the greatest extent, the differences caused by factors such as smoking, gender, weight

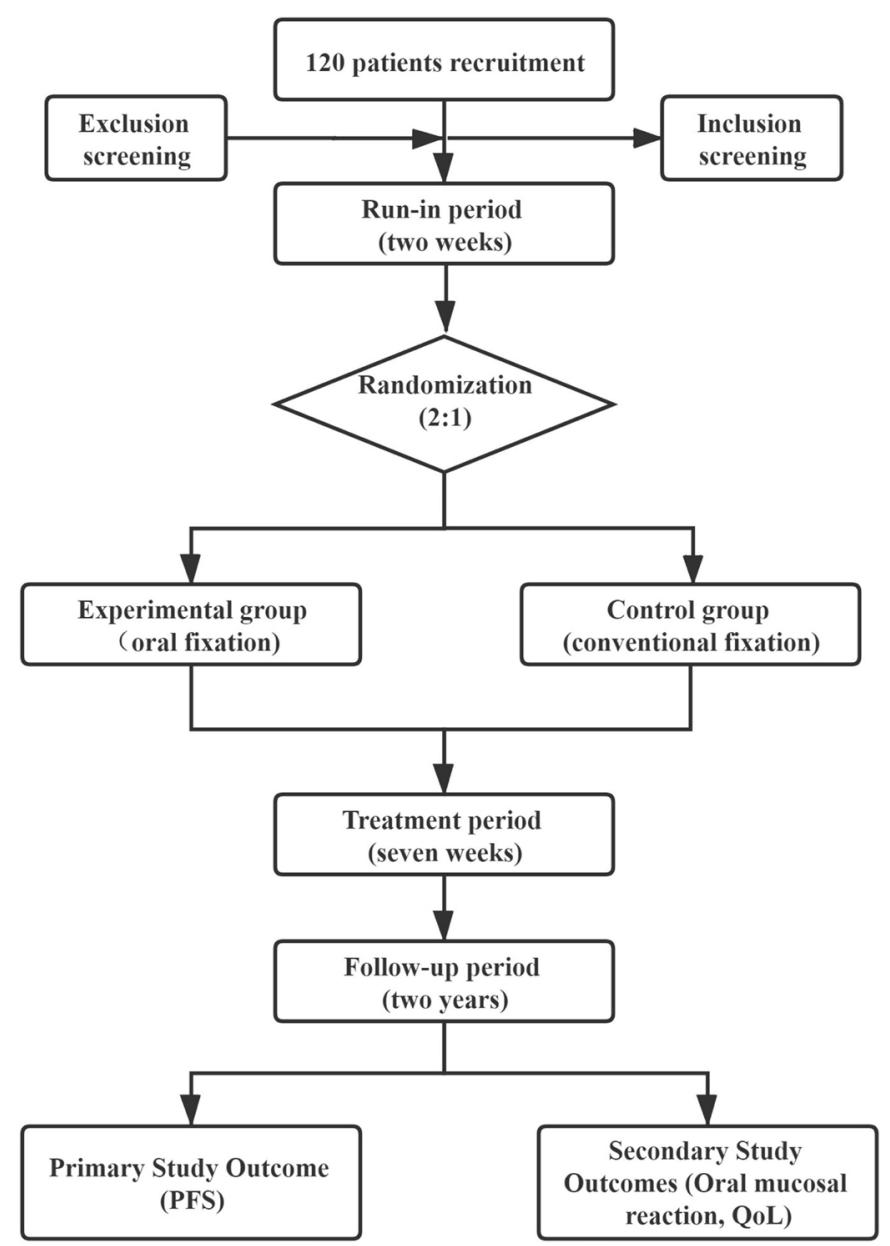

Figure 1 Flow chart representing the study procedures. PFS, progression-free survival time; QOL, quality of life.

loss, geographical factors, economic status, education level and other factors, and to avoid to the greatest extent, the baseline influence.

\section{ETHICS AND DISSEMINATION \\ Ethical considerations}

This code of ethics has been approved by the Medical Ethics Committee of Liaoning Provincial Cancer Hospital, number 20210131X.

\section{Adverse event report}

The main investigators will be notified as soon as possible of any adverse events during the study. Researchers and doctors will work together to determine their treatment plan as soon as possible. Serious adverse events will be reported to the main responsible person, the Ethics Committee and the relevant administrative departments will attend to them within a day.

\section{Suspension of clinical trials standards}

The clinical trial will be suspended if the patient fails to complete the relevant examination, or evaluation at follow-up within the agreed time. Alternatively, if the study 
cannot be suspended, that specific part of the patient's clinical trial will be suspended.

\section{Data monitoring}

The data monitoring committee (DMC) is composed of staff from the Data and Information Department of Liaoning Cancer Hospital. The DMC is responsible for independently reviewing and evaluating ongoing clinical trials to assess evolving risks to subject safety.

\section{Quality assurance of data quality and protocol adherence}

The fixed staff is responsible for data entry, coding groups 1 and 2 according to the treatment plan, and setting passwords for the database to protect the privacy of patient data. Data quality is controlled by the data quality Supervision Committee. The truthfulness and accuracy of the data are ensured to the maximum extent, and obviously wrong data can be eliminated. In addition, the compliance with the trial protocol is evaluated every 2 weeks by YS's team. Irregular protocol implementation can be corrected in a timely manner.

\section{Patient confidentiality}

We will do our best to maintain privacy and are determined to avoid the exposure of patients' personal information. In collecting, sharing and using participants' personal information and related data, all staff members follow the principle of confidentiality and do not disclose or disseminate patients' personal information.

\section{Dissemination}

The clinical trial data will be analysed and released as soon as possible. Before our team publishes the report, individual participants will not be able to publish patient data directly related to the trial. Our team will obtain the final data set, form a writing committee, and publish the test results at regional and national conferences. The final results will be presented at a scientific conference and published in a peer-reviewed journal in accordance with the journal's guidelines.

\section{DISCUSSION}

As far as we know, no project has been established to implement this type of research before. Its value will lie in its exploration of the efficacy of specific types of fixed and parameter collection devices during radiotherapy, and in providing data to support the development of key treatment norms and guidelines. ${ }^{29}{ }^{30}$ It will also provide a reference on which to base the choice of oral fixation devices for patients with other types of head and neck tumours. The design of this study will be based on the patented products of this project group: oral fixation and parameter acquisition device and TOMO technology, and the clinical application and transformation of scientific research results to provide new ideas for improving the prognosis of patients with head and neck tumours. ${ }^{31}$ It is expected to produce high-quality research results and greatly benefit patients. During the implementation of the study, individual differences in the actual conditions of the patients, the cooperation of the patients or other factors may affect the baseline level. In this case, our use of the $\chi^{2}$ test, combined with A propensity matching analysis, will eliminate to the greatest extent, individual differences and avoid baseline influence. We will collect enough statistical data to ensure the smooth progress of the experiment. During the research process, we will continue to consider additional details to ensure the smooth implementation of the study.

\section{Author affiliations}

${ }^{1}$ Department of Radiotherapy, Cancer Hospital of China Medical University, Shenyang, Liaoning, China

${ }^{2}$ China Medical University, Cancer Hospital of China Medical University, Shenyang, Liaoning, China

${ }^{3}$ Department of Information Management, Cancer Hospital of China Medical University, Shenyang, Liaoning, China

${ }^{4}$ Department of Pathology, Cancer Hospital of China Medical University, Shenyang, Liaoning, China

${ }^{5}$ Department of Radiotherapy Physics, Cancer Hospital of China Medical University, Shenyang, Liaoning, China

Acknowledgements We sincerely thank all research participants for taking the time to participate in this research.

Contributors YS conducted the conceptual design and revision of clinical trials. XZ wrote this article. TW is responsible for applying for and obtaining the approval of the ethics committee to complete the clinical trial registration; XL provides technical guidance, YS, XZ, TW and XL are first-line clinicians who are responsible for the screening and patient selection of patients who meet the requirements for entry into the clinical trial Join the group work. XX is responsible for inputting patient information and data, data collation and follow-up work. CYW is a statistician who is responsible for guiding data collection, management and analysis. LH is in charge of the physics room and strictly controls the quality of the patient's radiotherapy process. Pathologist BH provided pathology report guarantee for patients in clinical trials. All authors approved the final manuscript.

Funding This work will be supported by the Cancer Research Programme of National Cancer Centre (NCC2017A08) and Shenyang Major Scientific Research Projects (No. 191124090).

Competing interests None declared.

Patient consent for publication Not applicable.

Provenance and peer review Not commissioned; externally peer reviewed.

Supplemental material This content has been supplied by the author(s). It has not been vetted by BMJ Publishing Group Limited (BMJ) and may not have been peer-reviewed. Any opinions or recommendations discussed are solely those of the author(s) and are not endorsed by BMJ. BMJ disclaims all liability and responsibility arising from any reliance placed on the content. Where the content includes any translated material, BMJ does not warrant the accuracy and reliability of the translations (including but not limited to local regulations, clinical guidelines, terminology, drug names and drug dosages), and is not responsible for any error and/or omissions arising from translation and adaptation or otherwise.

Open access This is an open access article distributed in accordance with the Creative Commons Attribution Non Commercial (CC BY-NC 4.0) license, which permits others to distribute, remix, adapt, build upon this work non-commercially, and license their derivative works on different terms, provided the original work is properly cited, appropriate credit is given, any changes made indicated, and the use is non-commercial. See: http://creativecommons.org/licenses/by-nc/4.0/.

ORCID iD

Xiaofang Zhang http://orcid.org/0000-0003-0776-6327

\section{REFERENCES}

1 Yang J, Guo Y, Seo W, et al. Targeting cellular metabolism to reduce head and neck cancer growth. Sci Rep 2019;9:4995. 
2 Leung WS, Wu VWC, Liu CYW, et al. A dosimetric comparison of the use of equally spaced beam (ESb), beam angle optimization (BAO), and volumetric modulated Arc therapy (VMAT) in head and neck cancers treated by intensity modulated radiotherapy. J App/ Clin Med Phys 2019;20:121-30.

3 Xiang M, Chang DT, Pollom EL. Second cancer risk after primary cancer treatment with three-dimensional conformal, intensity-modulated, or proton beam radiation therapy. Cancer 2020;126:3560-8.

4 Uhl M, Sterzing F, Habl G, et al. Breast cancer and funnel chest. comparing helical tomotherapy and three-dimensional conformal radiotherapy with regard to the shape of pectus excavatum. Strahlenther Onkol 2012;188:127-35.

5 Lee $\mathrm{H}-\mathrm{H}$, Chen $\mathrm{C}-\mathrm{H}$, Luo K-H, et al. Five-Year survival outcomes of intensity-modulated radiotherapy with simultaneous integrated boost (IMRT-SIB) using forward IMRT or tomotherapy for breast cancer. Sci Rep 2020;10:4342.

6 Li J, Zhao Z, Wu X, et al. Bevacizumab plus cisplatin and helical tomotherapy in treatment of locally advanced nasopharyngeal carcinoma. Onco Targets Ther 2015;8:1315-9.

7 Lee J, Kim E, Kim N, et al. Practical aspects of the application of helical tomotherapy for craniospinal irradiation. Sci Rep 2021:11:6120.

8 Miura K, Kurosaki H, Utsumi N, et al. Use of a Head-Tilting baseplate during tomotherapy to shorten the irradiation time and protect the hippocampus and lens in hippocampal Sparing-Whole brain radiotherapy. Technol Cancer Res Treat 2021;20:153303382098682.

9 Chen G-P, Tai A, Puckett L, et al. Clinical implementation and initial experience of real-time motion tracking with jaws and Multileaf Collimator during helical tomotherapy delivery. Pract Radiat Oncol 2021:11:e486-95.

10 Yang C, Gong G, Jin E, et al. Topical application of honey in the management of chemo/radiotherapy-induced oral mucositis: a systematic review and network meta-analysis. Int J Nurs Stud 2019;89:80-7.

11 Peralta-Mamani M, da Silva BM, da Silva Pinto AC, et al. LowLevel laser therapy dosimetry most used for oral mucositis due to radiotherapy for head and neck cancer: a systematic review and meta-analysis. Crit Rev Oncol Hematol 2019;138:14-23.

12 Kawashita $\mathrm{Y}$, Koyama $\mathrm{Y}$, Kurita $\mathrm{H}$, et al. Effectiveness of a comprehensive oral management protocol for the prevention of severe oral mucositis in patients receiving radiotherapy with or without chemotherapy for oral cancer: a multicentre phase II, randomized controlled trial. Int J Oral Maxillofac Surg 2019;48:857-64.

13 Teng F, Fan W, Luo Y, et al. A risk prediction model by LASSO for radiation-induced xerostomia in patients with nasopharyngeal carcinoma treated with comprehensive salivary Gland-Sparing helical tomotherapy technique. Front Oncol 2021;11:633556.

14 Hsieh C-H, Shueng P-W, Wang L-Y, et al. Single-Institute clinical experiences using Whole-Field simultaneous integrated boost (Sib) intensity-modulated radiotherapy (IMRT) and sequential IMRT in postoperative patients with oral cavity cancer (OCC). Cancer Control 2020;27:107327482090470.

15 Zhang J, Jiang D, Liu H, et al. Analyzation of the local confidence limits for IMRT and VMAT based on AAPM TG119 report. Med Dosim 2020;45:66-72.
16 Nishimura $\mathrm{Y}$, Ishikura S, Shibata T, et al. A phase II study of adaptive two-step intensity-modulated radiation therapy (IMRT) with chemotherapy for loco-regionally advanced nasopharyngeal cancer (JCOG1015). Int J Clin Oncol 2020;25:1250-9.

17 Li C, Wang X, Wang L, et al. Clinical practice and outcome of radiotherapy for advanced esophageal squamous cell carcinoma between 2002 and 2018 in China: the multi-center 3JECROG survey. Acta Oncol 2021;60:1-8.

18 Mascha EJ, Vetter TR. Significance, errors, power, and sample size: the blocking and tackling of statistics. Anesth Analg 2018;126:691-8.

19 Kelcey B, Spybrook J, Dong N. Sample size planning for clusterrandomized interventions probing multilevel mediation. Prev Sci 2019;20:407-18.

20 Alotaibi F, Pelletier A, Gagnon J-F, et al. Prodromal marker progression in idiopathic rapid eye movement sleep behavior disorder: sample size for clinical trials. Mov Disord 2019;34:1914-9.

21 Zou R, Yuan J-J, Li Q, et al. The clinical outcomes and toxicities of induction chemotherapy followed by concurrent chemoradiotherapy plus adjuvant chemotherapy in locoregionally advanced nasopharyngeal carcinoma. Front Oncol 2020;10:619625.

22 Cai B, Meng L, Mo J, et al. Induction chemotherapy plus simultaneous modulated accelerated radiation therapy in nonoperative hypopharyngeal and supraglottic laryngeal squamous cell carcinoma: long-term outcome of a prospective phase 2 study. Front Oncol 2021;11:637978.

23 Lai C-H, Chen W-C, Fang C-C, et al. Survival-Weighted health profiles in nasopharyngeal cancer patients. Front Oncol 2021;11:635667.

24 Chen AC, Loiselle A, Zhou L, et al. Localization of peripheral pulmonary lesions using a method of computed TomographyAnatomic correlation and radial probe endobronchial ultrasound confirmation. Ann Am Thorac Soc 2016;13:1586-92.

25 Czarnecki CA, Einsiedel PF, Phal PM, et al. Dynamic CT for parathyroid adenoma detection: how does radiation dose compare with nuclear medicine? AJR Am J Roentgenol 2018;210:1118-22.

26 Huang S-F, Lin J-C, Shiau A-C, et al. Optimal tumor coverage with different beam energies by IMRT, VMAT and TOMO: effects on patients with proximal gastric cancer. Medicine 2020;99:e23328.

27 Hong JH, Kim Y-S, Lee S-W, et al. High-Dose thoracic re-irradiation of lung cancer using highly conformal radiotherapy is effective with acceptable toxicity. Cancer Res Treat 2019;51:1156-66.

28 Zhuang Q-Y, Li J-L, Lin F-F, et al. High biologically effective dose radiotherapy for brain metastases may improve survival and decrease risk for local relapse among patients with small-cell lung cancer: a Propensity-Matching analysis. Cancer Control 2020;27:107327482093628

29 Mitsuyoshi T, Matsuo Y, Shintani T, et al. Pilot study of the safety and efficacy of dose escalation in stereotactic body radiotherapy for peripheral lung tumors. Clin Lung Cancer 2018;19:e287-96.

30 Abravan A, Faivre-Finn C, Kennedy J, et al. Radiotherapy-Related lymphopenia affects overall survival in patients with lung cancer. $J$ Thorac Oncol 2020;15:1624-35.

31 Kitamori H, Sumida I, Tsujimoto T, et al. Evaluation of mouthpiece fixation devices for head and neck radiotherapy patients fabricated in PolyJet photopolymer by a 3D printer. Phys Med 2019;58:90-8. 